\title{
Human cancer cell line microRNAs associated with in vitro sensitivity to paclitaxel
}

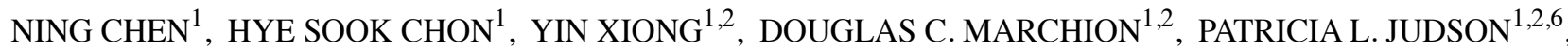 \\ ARDESHIR HAKAM $^{3,6}$, JESUS GONZALEZ-BOSQUET ${ }^{1,6}$, JENNIFER PERMUTH-WEY ${ }^{4}$, ROBERT M. WENHAM ${ }^{1,2,6}$, \\ SACHIN M. APTE ${ }^{1,6}$, JIN Q. CHENG ${ }^{5}$, THOMAS A. SELLERS ${ }^{4}$ and JOHNATHAN M. LANCASTER ${ }^{1,2,6}$ \\ ${ }^{1}$ Department of Women's Oncology, ${ }^{2}$ Experimental Therapeutics Program, and \\ Departments of ${ }^{3}$ Anatomic Pathology, ${ }^{4}$ Cancer Epidemiology, ${ }^{5}$ Molecular Oncology, and ${ }^{6}$ Oncologic Sciences, \\ H. Lee Moffitt Cancer Center and Research Institute, Tampa, FL 33612, USA
}

Received July 2, 2013; Accepted August 16, 2013

DOI: 10.3892/or.2013.2847

\begin{abstract}
Paclitaxel is a mainstay of treatment for many solid tumors, and frequently, clinical outcome is influenced by paclitaxel sensitivity. Despite this, ourunderstanding of the molecular basis of paclitaxel response is incomplete. Recently, it has been shown that microRNAs (miRNAs) influence messenger RNA (mRNA) transcriptional control and can contribute to human carcinogenesis. In the present study, our objective was to identify miRNAs associated with cancer cell line response to paclitaxel and to evaluate these miRNAs as therapeutic targets to increase paclitaxel sensitivity. We measured the expression of 335 unique miRNAs in 40 human cancer cell lines selected from the NCI panel. We then integrated miRNA expression data with publicly available paclitaxel-sensitivity $\left(\mathrm{GI}_{50}\right)$ data for each of the 40 cell lines to identify miRNAs associated with paclitaxel sensitivity. Ovarian cancer cell lines with differential miRNA expression and paclitaxel sensitivity were transiently transfected with miRNA precursors and inhibitors, and the effects on in vitro cell paclitaxel sensitivity were evaluated. Pearson's correlation identified 2 miRNAs (miR-367 and miR-30a-5p) associated with the NCI40 cell line in vitro paclitaxel response $(\mathrm{P}<0.0003)$. Ovarian cancer cells were selected based on the association between paclitaxel sensitivity and miR-367/miR-30a-5p expression. Overexpression of miR-367 in the paclitaxel-sensitive cells [PA1; $\mathrm{IC}_{50}, 1.69 \mathrm{nM}$, high miR-367 (2.997), low miR-30a-5p (-0.323)] further increased paclitaxel sensitivity, whereas miR-367 depletion decreased paclitaxel sensitivity. In contrast, overexpression and depletion of miR-30a-5p in the paclitaxel-resistant cells [OVCAR4; $\mathrm{IC}_{50}, 17.8 \mathrm{nM}$, low miR-367 (-0.640), high miR-30a-5p (3.270)] decreased and increased paclitaxel sensitivity, respectively. We
\end{abstract}

Correspondence to: Dr Johnathan M. Lancaster, Department of Women's Oncology, H. Lee Moffitt Cancer Center and Research Institute, 12902 Magnolia Drive, Tampa, FL 33612, USA

E-mail: johnathan.lancaster@moffitt.org

Key words: cancer, chemosensitivity, microRNA, NCI60 identified and successfully targeted miRNAs associated with human cancer cell line response to paclitaxel. Our strategy of integrating in vitro miRNA expression and drug sensitivity data may not only aid in the characterization of determinants of drug response but also in the identification of novel therapeutic targets to increase activity of existing therapeutics.

\section{Introduction}

Paclitaxel is a plant alkaloid that was developed from the bark of the Pacific yew tree, Taxus brevifolia (1). Paclitaxel is a taxane that stabilizes and disrupts microtubules required for cell division, resulting in cell death $(2,3)$. Despite extensive clinical use in the treatment of patients with lung, ovarian and breast cancer, long-term survival is frequently compromised by the development of paclitaxel resistance, for which the molecular basis remains to be fully delineated. microRNAs (miRNAs) are non-coding, 21-25 nucleotide regulatory RNAs that affect the stability and/or translational efficiency of messenger RNA (mRNAs) (4). Thousands of miRNAs are predicted to exist in the human genome (5) of which 1,100 human miRNAs have been identified, collectively targeting more than 19,000 human genes (www.microRNA.org). Deregulation of miRNAs has been implicated in the development of many types of human cancers $(6,7)$, suggesting that some miRNAs function as oncogenes or tumor suppressors $(8,9)$. It has been reported that loss of let7 may influence the development of lung cancer as it negatively regulates let60/RAS (10), whereas miRs-34a-c may play an important role in the tumor-suppressor function of p53 $(11,12)$ and miR-181a was found to be related to a morphological subclass of acute myeloid leukemia (13). Some studies have suggested that miRNAs may also influence chemosensitivity (14-16). It has been shown that miR-221/222 overexpression reduces $\mathrm{p} 27^{\mathrm{Kip} 1}$ levels and induces tamoxifen resistance due to cell cycle inhibition (17), whereas inhibition of miR-21 increases apoptosis in lung adenocarcinoma epithelial cell line A549 after NSC 265450 (nogamycin) and NSC 670550 treatment by downregulating $\mathrm{Bcl} 2$ protein (14).

In the present study, we integrated miRNA data for lung, colon, breast, ovarian, kidney, skin (melanoma), prostate, central nervous system (CNS), and hematologic (leukemia) 
Table I. Cancer cell lines subjected to miRNA expression analyses.

Origin of cancer tissue

Cell lines

\begin{tabular}{ll}
\hline Lung & NCI-H460, NCI-H522, NCI-H322M, HOP62, A549, EKVX, MALME-3M, NCI-H226 \\
Colon & HT29, HCT-116, SE-620, HCT-15, HCC2998, COLO205 \\
Breast & HS-578T, NCI/ADR-RES \\
Ovarian & OVCAR8, OVCAR4 \\
Renal & ACHN, SN-12C, 786-O, CAKI-1, UO-31, TK-10, A498 \\
Melanoma & SK-MEL-28, UACC-257, M14, UACC-62, SK-MEL-2, LOX-IMVI \\
Prostate & DU-145, PC-3 \\
CNS & SF-295, SF-539, SNB-75, U251 \\
Leukemia & HL-60, RPMI8226, K562
\end{tabular}

cancer cell lines with $\mathrm{GI}_{50}$ paclitaxel-sensitivity data in an effort to identify miRNAs associated with paclitaxel response. Furthermore, we evaluated the effect of in vitro targeted modulation of these miRNAs on paclitaxel sensitivity.

\section{Materials and methods}

Cell culture and reagents. A subset of 40 of the NCI60 cancer cell line panel was obtained from the National Cancer Institute (NCI) Developmental Therapeutics Program (Table I). Ovarian cancer (OVCA) cell lines in addition to those on the NCI60 panel were obtained from the American Type Culture Collection (ATCC, Manassas, VA, USA; CAOV3, OV90, OVCAR3 and SKOV3), the European Collection of Cell Cultures (Salisbury, UK; A2780CP and A2780S), Kyoto University (Kyoto, Japan; M41, M41CSR, Tyknu, and TyknuCisR), or as kind gifts from Dr Patricia Kruk, Department of Pathology, College of Medicine, University of South Florida, Tampa, FL, and Susan Murphy, Department of OBGYN/Division of Gynecologic Oncology, Duke University, Durham, NC (HeyA8, IGR-OV1, IMCC3, IMCC5, MCAS, OVCA420, OVCA429, OVCA432, OVCA433, FUOV1, PA1, PEO1, PEO4, T8, TOV-112D, TOV-21-G, Dov13, OVCAR10, OVCAR8, OVCAR5, OVCAR4 and OVCAR2). Human stem cell lines (H9) were obtained from WiCell (Madison, WI, USA). All cancer cell lines were cultured in RPMI-1640 medium supplemented with $1 \%$ non-essential amino acids, $1 \%$ sodium pyruvate and $10 \%$ fetal bovine serum. H9 cells were cultured according to the manufacturer's protocol. Cells were cultured for 2-3 passages before experimentation. Paclitaxel was obtained from Sequoia Research Products Ltd., (Oxfordshire, UK), dissolved in DMSO at a concentration of $100 \mathrm{mM}$ and stored at $-20^{\circ} \mathrm{C}$.

miRNA extraction and expression profiling. Total RNA was extracted from $1 \times 10^{6}$ log-phase cells using the mirVana miRNA isolation kit (Life Technologies) according to the manufacturer's instructions. The yield and quality of total RNA for each cell line were determined using an Agilent Bioanalyzer. The RNA integrity number (RIN) of all samples was in a range of 8-10 and no genomic DNA contamination was detected. Total RNA (10 $\mu \mathrm{g})$ from each cell line was then subjected to miRNA expression profiling. The cell line samples were co-hybridized to printed arrays that contained a 562 Ambion mirVana miRNA probe set (Ambion, Austin TX, USA) and 632 of Invitrogen's NCode Multi-Species miRNA probes (Invitrogen, Carlsbad, CA, USA), which contain $>800$ unique human miRNAs. The hybridized arrays were scanned on a GenePix 4000B scanner, and expression data were generated using the GenePix Pro software (Molecular Devices, Sunnyvale, CA, USA).

Transfection of miRNA. Cell lines were transfected with pre-miR-367, pre-miR-30a-5p, anti-miR-367 or anti-miR-30a-5p (Life Technologies) using siPORT NeoFX transfection reagent, according to the manufacturer's protocol. The concentrations of miRNA and the transfection reagent were optimized by the manufacturer's recommendations prior to experimentation. Log-phase cells ( $\sim 60 \%$ confluence) were incubated with $6.25 \mu \mathrm{M}$ precursor or inhibitor miRNAs in the presence of $50 \mathrm{nM}$ transfection reagent for $4 \mathrm{~h}$. pre-miR negative control 1 precursor and anti-miR negative control 1 were used as controls for miRNA overexpression and depletion experiments, respectively. Control miRNAs were transfected under the same condition as the experimental miRNAs. Both control miRNAs have mechanisms similar to the pre-miRNA precursors and anti-miRNA inhibitors but have no observed effects on known miRNA functions.

Comparative $C_{T} R T$-PCR. Real-time comparative $\mathrm{C}_{\mathrm{T}}$ RT-PCR was used to determine relative miRNA levels. miRNAs were converted into cDNA using miRNA sequence-specific primers and the TaqMan ${ }^{\circledR}$ microRNA reverse transcription (Applied Biosystems). Total RNA (10 ng) was used for each $15-\mu 1$ reverse transcription reaction. Comparative $C_{T}$ RT-PCR was performed on the Applied Biosystems StepOne RealTime PCR system, according to the manufacturer's protocols. Briefly, $1.33 \mu 1$ of miRNA-specific cDNA was combined with sequence-specific TaqMan miRNA assays and TaqMan Universal PCR Master Mix, no AmpErase UNG in a total reaction volume of $20 \mu \mathrm{l}$. RNU44 was used as the endogenous miRNA control for normalization. RNU44 was considered a qualified candidate of endogenous control based on preliminary experimentation (data not shown). miRNA expression levels were analyzed using StepOne Real-Time PCR instrument software. 
Growth inhibition assay. Cells were seeded in 96-well plates (Perkin-Elmer) at a density of $6 \times 10^{4}$ cells $/ \mathrm{ml}$ and incubated overnight at $37^{\circ} \mathrm{C}$. Cells were incubated with the indicated concentrations of paclitaxel for $72 \mathrm{~h}$, and cell viability was assessed using the CellTiter-Glo ${ }^{\mathrm{TM}}$ luminescent cell viability assay kit (MTS kit; Promega). Luminescence was recorded using Wallace Victor2 ${ }^{\text {TM }} 1420$ Multilabel Counter (PerkinElmer). Wells containing medium without cells were used to obtain background luminescence. All experimental wells and controls were set up in triplet. Paclitaxel $\log _{10}\left(\mathrm{IC}_{50}\right)$ values for transfected cell lines were calculated using the sigmoidal dose-response (variable slope) curve equation (Prism 5). Dose-curve graphs were generated using GraphPad (Prism 5). Cells cultured with transfected medium without miRNA precursors or inhibitors were used as controls. The effects of increased pre-miR and anti-miR levels due to transfection on cell viability were assessed $48 \mathrm{~h}$ post-transfection using MTS assays.

Statistical analysis. Two color spotted array data for miRNA levels were generated from the GenePix Pro software. Background subtraction (18) and Loess normalization (19) were performed using Limma software. Replicate probe sets were averaged by their design (Ambion or Invitrogen). Processed data were then analyzed using SAM (significance analysis of microarrays) software (20). Missing values were imputed with SAM's nearest neighbor imputer, where $k$ was set to 10. For each drug, Pearson's correlation test was used to identify those miRNAs with expression values associated with sensitivity measured by $\mathrm{GI}_{50}$.

Pathway analysis. The miRanda database was used to identify the mRNA targets of miRNAs found to be associated with in vitro sensitivity to chemotherapy. The identified mRNA targets were subjected to GeneGo MetaCore analysis to determine biological signaling pathway representation. $\mathrm{P}<0.05$ represented statistical significance of the association between the mRNA targets of the miRNAs and the biological pathways.

\section{Results}

Correlation of miRNA expression and paclitaxel sensitivity/resistance. Paclitaxel sensitivity $\left(\mathrm{GI}_{50}\right)$ data for the subset of 40 cancer cell lines of the NCI60 cell panel (3 leukemia, 6 melanoma, 8 non-small cell lung, 6 colon, 4 central nervous system, 2 ovarian, 7 renal, 2 prostate and 2 breast cancer cell lines) was obtained from NCI Website (http://dtp.nci.nih.gov/ dtpstandard/cancerscreeningdata/index.jsp). Based on miRNA expression and $\mathrm{GI}_{50}$ data, Pearson's correlation test identified 35 miRNAs associated with in vitro paclitaxel sensitivity $(\mathrm{P}<0.05)$. miR-367 and miR-30a-5p demonstrated the highest level of statistical significance in association with sensitivity to paclitaxel $(\mathrm{P}<0.0003)$ (Table II).

Selection of cell lines. The OVCA cell lines PA1 and OVCAR4 were selected for further evaluation based on associations between paclitaxel sensitivity and miR-367/miR-30a-5p expression. An evaluation of the OVCA cell lines showed PA1 to have a higher relative expression value of miR-367 (2.997), a lower relative expression value of miR-30a-5p (-0.32), and
Table II. microRNAs associated with paclitaxel sensitivity.

\begin{tabular}{|c|c|c|}
\hline miRNA & P-value & Up/down \\
\hline hsa_miR_367 & 0.00022 & Down \\
\hline hsa_miR_30a_5p & 0.00028 & Up \\
\hline hsa_miR_141 & 0.00115 & Down \\
\hline hsa_miR_30a_3p & 0.00306 & Up \\
\hline hsa_miR_516_3p & 0.00318 & Up \\
\hline hsa_miR_377 & 0.00350 & Down \\
\hline hsa_miR_134 & 0.00371 & Up \\
\hline hsa_miR_142_5p & 0.00767 & Down \\
\hline hsa_let_7e & 0.01000 & Up \\
\hline hsa_miR_29c & 0.01017 & Down \\
\hline hsa_miR_218 & 0.01105 & Down \\
\hline hsa_miR_17_3p & 0.01123 & Down \\
\hline hsa_miR_17_5p & 0.01288 & Down \\
\hline hsa_miR_130a & 0.01289 & Up \\
\hline hsa_miR_195 & 0.01939 & Down \\
\hline hsa_miR_99b & 0.02003 & Up \\
\hline hsa_miR_338 & 0.02508 & $\mathrm{Up}$ \\
\hline hsa_miR_106a & 0.02611 & Down \\
\hline hsa_miR_193b & 0.02720 & Up \\
\hline hsa_miR_515_3p & 0.02884 & Up \\
\hline hsa_miR_374 & 0.02889 & Up \\
\hline hsa_miR_125a & 0.02993 & Up \\
\hline hsa_miR_192 & 0.03023 & Down \\
\hline hsa_miR_30c & 0.03039 & Up \\
\hline hsa_miR_95 & 0.03684 & Down \\
\hline hsa_miR_452_AS & 0.03703 & Down \\
\hline hsa_miR_489 & 0.03774 & Up \\
\hline hsa_miR_32 & 0.03897 & Down \\
\hline hsa_miR_373* & 0.03938 & Up \\
\hline hsa_miR_130b & 0.04136 & Down \\
\hline hsa_miR_19b & 0.04385 & Down \\
\hline hsa_miR_126 & 0.04543 & Down \\
\hline hsa_miR_148a & 0.04650 & Down \\
\hline hsa_miR_376b & 0.04835 & Up \\
\hline hsa_miR_7 & 0.04835 & Up \\
\hline
\end{tabular}

Positive correlation, up; negative correlation, down.

relative sensitivity to paclitaxel-induced cell growth arrest $\left(\mathrm{IC}_{50}, 1.69 \mathrm{nM}\right)$. In contrast, OVCAR4 showed a lower relative expression value of miR-367 (-0.64), a higher relative expression values of miR-30a-5p (3.27), and was more resistant to paclitaxel $\left(\mathrm{IC}_{50}, 17.8 \mathrm{nM}\right)$. The differential expression of miR-367 and miR-30a-5p in PA1 and OVCAR4 cells was confirmed by quantitative RT-PCR (Fig. 1). As a reference, miRNA expression was compared to embryonic stem cells, which reportedly express miR-367 in the early stage and reduced miR-367 expression in the late stage (21). Embryonic stems cells were defined as early vs. late stage based on culture 

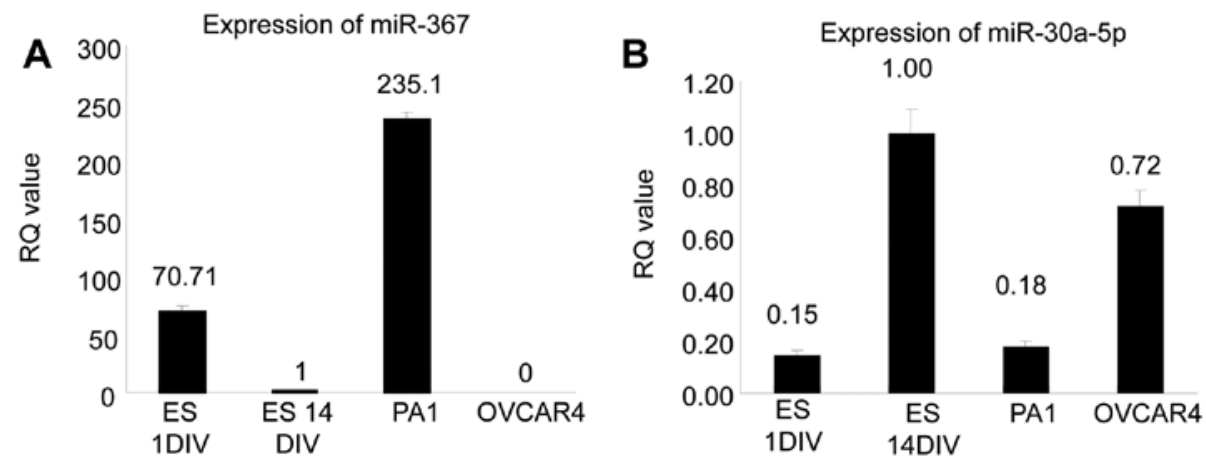

Figure 1. Differential expression of miR-367 and miR-30a-5p by quantitative RT-PCR. The differential expression of (A) miR-367 and (B) miR-30a-5p was confirmed by RT-PCR in the ovarian cancer cell lines, PA1 and OVCAR4. Embryonic stem cells cultured for 1 day (early-stage, ES1DIV) and 14 days (latestage, ES14DIV) were used as controls for positive and negative miRNA expression, respectively. RNU44 was used as endogenous control. ES14DIV was used as the sample reference control. The value of the reference control was set as 1.
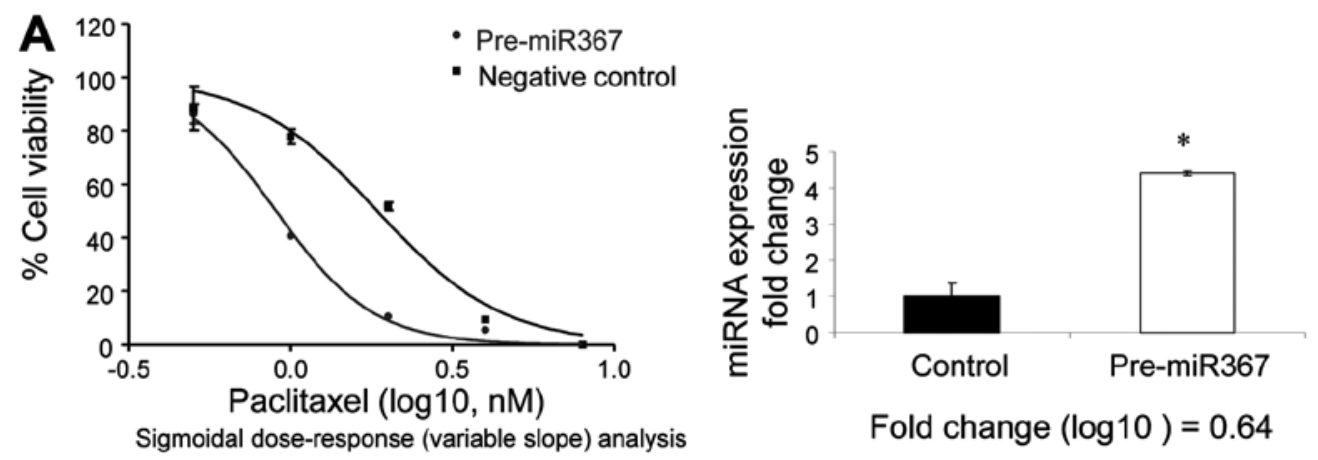

Fold change $(\log 10)=0.64$
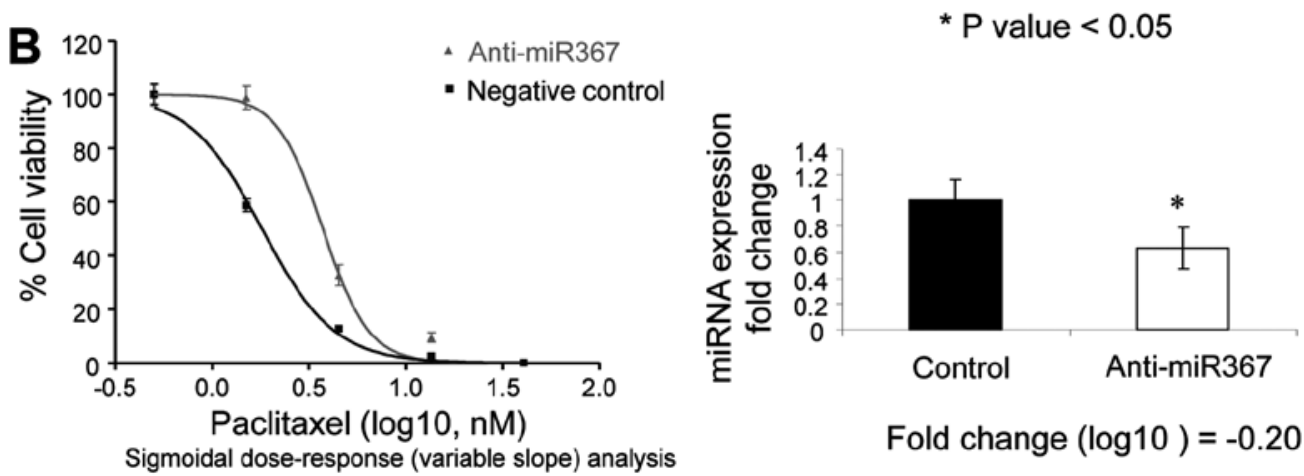

Figure 2. Modulation of miRNA expression affects paclitaxel sensitivity. PA1 cells (high miR-367 expression, low miR-30a-5p expression) were evaluated for paclitaxel-induced growth arrest at 72 and $48 \mathrm{~h}$ after transient transfection of (A) pre-miR-367 precursor miRNA and (B) anti-miR-367 inhibitor miRNA. Changes in miRNA levels were evaluated $48 \mathrm{~h}$ after transfection by comparative $\mathrm{C}_{\mathrm{T}}$ RT-PCR. The negative control was set as the reference sample and the fold-change as 1 . The endogenous control was RNU44. The fold-change before and after transfection was calculated by $2^{-\Delta \Delta C t}$.

duration of 1 (ES1DIV) vs. 14 (ES14DIV) days, respectively. As shown in Fig. 1A, using ES14DIV as the reference [relative expression $(\mathrm{RQ})=1]$, the relative expression (RQ value) of miR-367 PA1 cells was 235.1, compared to $\mathrm{RQ}=70.71$ for ES1DIV (positive control) and RQ=0 for OVCAR4 cells. In contrast, normalized to ES14DIV (RQ=1), OVCAR4 had the highest expression of miR-30a-5p with an $\mathrm{RQ}=0.72$, compared to $\mathrm{RQ}=0.18$ for PA1 cells (Fig. 1B).

Evaluation of miR-367 and miR-30a-5p as therapeutic targets. To determine the value of miR-367 and miR-30a-5p as therapeutic targets in OVCA cells, miR-367 was overexpressed or depleted in the paclitaxel-resistant cell line PA1 (high miR-367, low miR-30a-5p), through transient transfection of the miRNA precursor (pre-miR-367) and inhibitor (anti-miR-367), respectively. In contrast, the paclitaxel-sensitive OVCA cell line OVCAR4 (low miR-367, high miR-30a-5p) was transfected with the miR-30a-5p precursor (pre-miR-30a-5p) and inhibitor (anti-miR-30a-5p). Forty-eight hours after transfection, cells were incubated with increasing doses of paclitaxel for $72 \mathrm{~h}$ and evaluated for cell viability using the CellTiter-Glo ${ }^{\mathrm{TM}}$ luminescent assay.

Compared to the mock transfection controls, PA1 cells transfected with pre-miR-367 showed a 57\% decrease in cell survivability $48 \mathrm{~h}$ after transfection, whereas transfection of anti-miR-367 did not affect cell survival. Transfection 

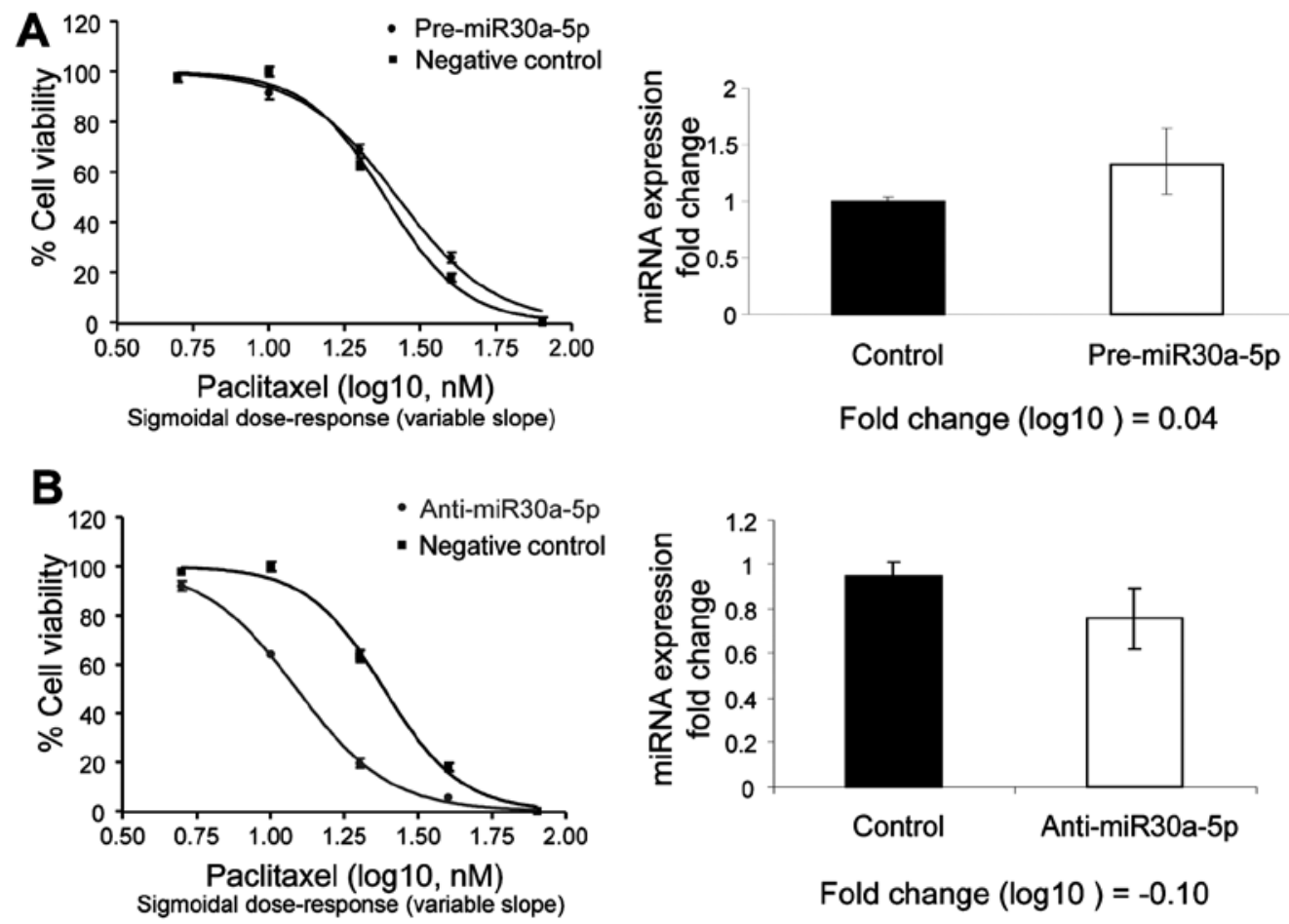

Fold change $(\log 10)=0.04$

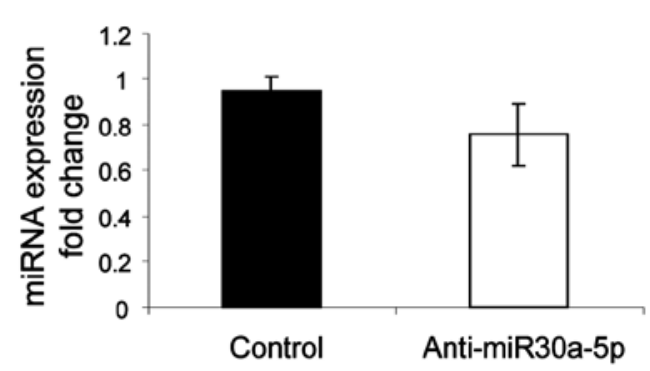

Fold change $(\log 10)=-0.10$

Figure 3. Modulation of miRNA expression affects paclitaxel sensitivity. OVCAR4 cells (low miR-367 expression, high miR-30a-5p expression) were evaluated for paclitaxel-induced growth arrest at 72 and $48 \mathrm{~h}$ after transient transfection of (A) pre-miR-30a-5p precursor miRNA and (B) anti-miR-30a-5p inhibitor miRNA. Non-targeting pre-miRNA and anti-miRNA negative controls were used as references. Changes in miRNA levels were evaluated $48 \mathrm{~h}$ after transfection by comparative $\mathrm{C}_{\mathrm{T}}$ RT-PCR. The negative control was set as the reference sample and the fold-change as 1 . The endogenous control was RNU44. The fold-change before and after transfection was calculated by $2^{-\Delta \Delta C t}$.

of pre-miR-30a-5p and anti-miR-30a-5p had no effect on the survivability of PA1 cells (data not shown). Despite the decrease in cell survival, transfection of pre-miR-367 in PA1 cells resulted in a $0.64 \log _{10}$-fold-change in miR-367 expression and an increase in paclitaxel sensitivity when compared to the precursor negative control (Fig. 2A). In contrast, transfection of PA1 cells with anti-miR-367 resulted in a $-0.2 \log _{10}$-foldchange and a decrease in paclitaxel sensitivity (Fig. 2B).

In the intrinsically paclitaxel-resistant cell line (OVCAR4), which has high relative expression of miR-30a-5p and low relative expression of miR-367, overexpression of the miR-30a-5p precursor (0.04 $\log _{10}$-fold change) slightly decreased paclitaxel-induced sensitivity, whereas depletion of miR-30a-5p (-0.10 $\log _{10}$-fold change) increased paclitaxel-induced growth arrest (Fig. 3).

Pathways involved in the deregulation of miRNAs. To evaluate the potential influence of miR-367 and miR-30-5p on various cellular processes, we identified the predicted mRNA target genes of these miRNAs using the miRanda database (22). This database hosts target sites for 1,100 human miRNAs and 16,228,619 predicted miRNA targets in 34,911 distinct 3'UTRs of 19,898 human genes. The miRanda database predicted 1,536 and 2,320 target genes associated with miR-367 and miR-30a-5p, respectively (mirSVR score $>-0.15$, mirSVR score ranged from -0.1 to -1.35 ) (20). These predicted miRNA targets were further analyzed for biologic signaling pathway representation using GeneGo MetaCore software. Pathway modeling identified 16 pathways represented among the predicted target genes for miR-367 $(\mathrm{P}<0.0001)$ and 20 path- ways were represented among the target genes for miR-30a $(\mathrm{P}<0.0001)$ (Table III).

\section{Discussion}

The efficacy of cancer treatment is frequently limited by intrinsic and acquired resistance to chemotherapy. Despite progress in delineating the molecular determinants of cancer chemo-response, a comprehensive understanding of the factors that underlie drug resistance remains elusive.

Evidence is accumulating to support a role for miRNAs in the development and progression of human cancer $(7,23,24)$. Moreover, recent data also suggest that miRNAs may influence cancer cell response to chemotherapy $(14,16,25)$ by mechanisms that may be both cancer cell-type or drug specific. Previous studies have shown that paclitaxel sensitivity may be associated with the expression of miR-200c in both ovarian (26) and gastric cancer (27), miR-148 in prostate cancer cells (28), miR-337, miR-34 and miR-135a in lung cancer (29-31), miR-22 in colon cancer (32), and miR-125b and miR-21 in breast cancer $(33,34)$.

In the present study, miRNA expression data integrated with publicly available chemosensitivity data for 40 human cancer cell lines (representing 9 different cancer cell types) identified 35 miRNAs to be associated with in vitro paclitaxel sensitivity $(\mathrm{P}<0.05)$. Two of these miRNAs, miR-367 and miR-30a-5p, were selected for further experimentation based on associations between paclitaxel sensitivity and miR-367/miR-30a-5p expression. The effects of miR-367 and miR-30a-5p expression on chemosensitivity were investigated 
Table III. miR-367/miR-30a-5p target gene-involved pathways $(\mathrm{P}<0.0001)$.

P-value

$1.84 \mathrm{E}-07$

$2.58 \mathrm{E}-07$

2.63E-07

$1.16 \mathrm{E}-06$

$2.44 \mathrm{E}-06$

2.54E-06

$1.01 \mathrm{E}-05$

$1.32 \mathrm{E}-05$

$1.53 \mathrm{E}-05$

2.09E-05

4.19E-05

4.19E-05

4.68E-05

$5.22 \mathrm{E}-05$

$6.08 \mathrm{E}-05$

9.64E-05

Neurophysiological process_ACM regulation of nerve impulse

miR30a-5p target gene-involved pathways $(\mathrm{P}<0.0001)$

Cytoskeleton remodeling_TGF, WNT and cytoskeletal remodeling

Cytoskeleton remodeling_Cytoskeleton remodeling

Cell adhesion_Ephrin signaling

Development_Thrombopoietin-regulated cell processes

Development_HGF signaling pathway

Development_WNT signaling pathway. Part 2

Muscle contraction_Regulation of eNOS activity in endothelial cells

Development_Regulation of epithelial-to-mesenchymal transition (EMT)

Apoptosis and survival_FAS signaling cascades

Development_Membrane-bound ESR1: interaction with growth factor signaling

Cardiac hypertrophy_NF-AT signaling in cardiac hypertrophy

Immune response_ETV3 affect on CSF1-promoted macrophage differentiation

Development_Role of IL-8 in angiogenesis

Development_Ligand-independent activation of ESR1 and ESR2

Cell adhesion_Chemokines and adhesion

Translation_Regulation of EIF4F activity

Apoptosis and survival_Caspase cascade

Development_PIP3 signaling in cardiac myocytes

PGE2 pathways in cancer

DNA damage_Role of SUMO in p53 regulation
8.72E-08

2.81E-07

$1.02 \mathrm{E}-06$

$1.02 \mathrm{E}-06$

$1.84 \mathrm{E}-06$

8.82E-06

$2.05 \mathrm{E}-05$

$2.05 \mathrm{E}-05$

2.12E-05

$2.12 \mathrm{E}-05$

2.5E-05

2.58E-05

2.7E-05

2.73E-05

4.13E-05

4.36E-05

4.75E-05

5.59E-05

6.61E-05

7.41E-05
Objects/networks

$14 / 15$

$12 / 38$

$13 / 45$

$12 / 43$

$9 / 25$

$12 / 46$

$12 / 52$

$11 / 45$

$12 / 54$

$10 / 39$

$10 / 42$

$10 / 42$

$11 / 51$

$10 / 43$

$13 / 71$

$10 / 46$

$25 / 111$

$23 / 102$

$14 / 45$

$14 / 45$

$14 / 47$

$14 / 53$

$15 / 64$

$15 / 64$

$12 / 43$

$12 / 43$

$15 / 65$

10/31

$14 / 58$

$12 / 44$

$19 / 100$

$13 / 53$

$10 / 33$

$12 / 47$

$13 / 55$

$7 / 17$ in OVCA cell lines shown to have differential expression of these miRNAs. The OVCA cell line PA1 was found to be relatively sensitive to paclitaxel-induced cell death and have relatively high expression of miR-367 and low expression of miR-30a-5p. In contrast, OVCAR4 cells were found to have almost no expression of miR-367 and relatively high expression of miR-30a-5p and were relatively resistant to paclitaxel. In PA1 cells, the overexpression and depletion of miR-367 increased the sensitivity and resistance of these cell lines to paclitaxel-induced growth arrest and cell death, respectively. In contrast, in OVCAR4 cells, an increase in miR-30a-5p expression was associated with decreased paclitaxel sensitivity, whereas a depletion of miR-30a-5p was associated with an increase in paclitaxel sensitivity. The mechanism by which these miRNAs affect chemosensitivity was not determined. However, miR-367, which belongs to the miR302 cluster, is only present in embryonic stem cells and is significantly decreased after cells differentiate (21). miR-302 and miR-367 
not only participate in the processes of maintaining cell selfrenewal and pluripotency in embryonic stem cells but are also overexpressed in various cancer cells $(21,35-38)$ and may play a role in chemosensitivity (39). Similarly, miR-30a-5p has been reported to be differentially expressed in various malignancies, including lung, thyroid, anaplastic and gastric cancer (40-44) and has been associated with survival of patients with cancer $(45,46)$.

Bioinformatic analyses of the predicted miR-367 target genes indicated that miR-367 may have an important regulatory role in the expression or activity of 16 biologic signaling pathways. Notably, the majority of these miR-367-influenced pathways, such as signal transduction/PKA signaling, signal transduction/AKT signaling, cAMP signaling, and apoptosis and survival/BAD phosphorylation, influence cell survival through regulating cell cycle and cell apoptosis and maintaining cell self-renewal and stemness (Table III), although the role of miR-367 in carcinogenesis and chemosensitivity is largely unknown.

miR-30a-5p was predicted to influence the expression of 20 biologic signaling pathways, several of which are also known to influence cellular survival, such as apoptosis and survival/FAS signaling cascades and apoptosis and survival/caspase cascade. However, the majority of pathways under the influence of miR30a-5p's appears to involve cytoskeletal remodeling and cell migration (Table III).

The present study demonstrated that the integration of miRNA expression data with existing chemosensitivity data from the NCI40 cell line set may provide insight into miRNAs that influence in vitro paclitaxel sensitivity. However, it should be acknowledged that such an approach may preferentially identify those miRNAs that were influential in determination of chemosensitivity across tumor types and may not identify those miRNAs that have a cancer-specific influence on the response to paclitaxel. Although data such as these provide an important contribution to our knowledge of the underpinnings of cancer cell response to therapeutic agents, it should be recognized that a comprehensive understanding of the biologic determinants of chemo-response will ultimately require us to incorporate information on additional variables such as DNA sequence and copy number, mRNA expression (vs. predicted mRNA targets), and protein levels and post-translational modifications. Our data contribute to the growing body of evidence suggesting that miRNAs have potential utility as personalized medicine biomarkers of cancer cell response to therapy and, moreover, may also represent viable therapeutic targets to increase cancer cell chemosensitivity.

\section{Acknowledgements}

This study was supported (in part) by Moffitt/USF Anna Valentine Fund and the Cancer Center Support Grant P30CA76292-14. We thank Rasa Hamilton (Moffitt Cancer Center) for her editorial assistance.

\section{References}

1. Wani MC, Taylor HL, Wall ME, Coggon P and McPhail AT: Plant antitumor agents. VI. The isolation and structure of Taxol, a novel antileukemic and antitumor agent from Taxus brevifolia. J Am Chem Soc 93: 2325-2327, 1971
2. Schiff PB and Horwitz SB: Taxol assembles tubulin in the absence of exogenous guanosine 5'-triphosphate or microtubuleassociated proteins. Biochemistry 20: 3247-3252, 1981.

3. Schiff PB and Horwitz SB: Taxol stabilizes microtubules in mouse fibroblast cells. Proc Natl Acad Sci USA 77: 1561-1565, 1980.

4. Lim LP, Lau NC, Garrett-Engele P, et al: Microarray analysis shows that some microRNAs downregulate large numbers of target mRNAs. Nature 433: 769-773, 2005.

5. Cummins JM and Velculescu VE: Implications of micro-RNA profiling for cancer diagnosis. Oncogene 25: 6220-6227, 2006.

6. He H, Jazdzewski K, Li W, et al: The role of microRNA genes in papillary thyroid carcinoma. Proc Natl Acad Sci USA 102: 19075-19080, 2005.

7. Lu J, Getz G, Miska EA, et al: MicroRNA expression profiles classify human cancers. Nature 435: 834-838, 2005.

8. Chen CZ: MicroRNAs as oncogenes and tumor suppressors. N Engl J Med 353: 1768-1771, 2005.

9. Hwang HW and Mendell JT: MicroRNAs in cell proliferation, cell death, and tumorigenesis. Br J Cancer 94: 776-780, 2006

10. Johnson SM, Grosshans H, Shingara J, et al: RAS is regulated by the let-7 microRNA family. Cell 120: 635-647, 2005.

11. Corney DC, Flesken-Nikitin A, Godwin AK, Wang W and Nikitin AY: MicroRNA-34b and MicroRNA-34c are targets of p53 and cooperate in control of cell proliferation and adhesionindependent growth. Cancer Res 67: 8433-8438, 2007.

12. Chang TC, Wentzel EA, Kent OA, et al: Transactivation of miR-34a by 53 broadly influences gene expression and promotes apoptosis. Mol Cell 26: 745-752, 2007.

13. Debernardi S, Skoulakis S, Molloy G, Chaplin T, Dixon-McIver A and Young BD: MicroRNA miR-181a correlates with morphological sub-class of acute myeloid leukaemia and the expression of its target genes in global genome-wide analysis. Leukemia 21: 912-916, 2007.

14. Blower PE, Chung JH, Verducci JS, et al: MicroRNAs modulate the chemosensitivity of tumor cells. Mol Cancer Ther 7: 1-9, 2008.

15. Zheng T, Wang J, Chen X and Liu L: Role of microRNA in anticancer drug resistance. Int J Cancer 126: 2-10, 2010.

16. Boren T, Xiong Y, Hakam A, et al: MicroRNAs and their target messenger RNAs associated with ovarian cancer response to chemotherapy. Gynecol Oncol 113: 249-255, 2009.

17. Zheng A, Kallio A and Harkonen P: Tamoxifen-induced rapid death of MCF-7 breast cancer cells is mediated via extracellularly signal-regulated kinase signaling and can be abrogated by estrogen. Endocrinology 148: 2764-2777, 2007.

18. Ritchie ME, Silver J, Oshlack A, et al: A comparison of background correction methods for two-colour microarrays. Bioinformatics 23: 2700-2707, 2007.

19. Smyth GK and Speed T: Normalization of cDNA microarray data. Methods 31: 265-273, 2003.

20. Tusher VG, Tibshirani R and Chu G: Significance analysis of microarrays applied to the ionizing radiation response. Proc Natl Acad Sci USA 98: 5116-5121, 2001.

21. Bar M, Wyman SK, Fritz BR, et al: MicroRNA discovery and profiling in human embryonic stem cells by deep sequencing of small RNA libraries. Stem Cells 26: 2496-2505, 2008.

22. Wang $X$ and El Naqa IM: Prediction of both conserved and nonconserved microRNA targets in animals. Bioinformatics 24: 325-332, 2008.

23. Sontheimer EJ and Carthew RW: Silence from within: endogenous siRNAs and miRNAs. Cell 122: 9-12, 2005.

24. O'Donnell KA, Wentzel EA,Zeller KI, Dang CV and Mendell JT: c-Myc-regulated microRNAs modulate E2F1 expression. Nature 435: 839-843, 2005.

25. Blower PE, Verducci JS, Lin S, et al: MicroRNA expression profiles for the NCI-60 cancer cell panel. Mol Cancer Ther 6: 1483-1491, 2007.

26. Cittelly DM, Dimitrova I, Howe EN, et al: Restoration of miR-200c to ovarian cancer reduces tumor burden and increases sensitivity to paclitaxel. Mol Cancer Ther 11: 2556-2565, 2012.

27. Chen Y, Zuo J, Liu Y, Gao H and Liu W: Inhibitory effects of miRNA-200c on chemotherapy-resistance and cell proliferation of gastric cancer SGC7901/DDP cells. Chin J Cancer 29: 1006-1011, 2010

28. Fujita Y, Kojima K, Ohhashi R, et al: MiR-148a attenuates paclitaxel resistance of hormone-refractory, drug-resistant prostate cancer PC 3 cells by regulating MSK1 expression. J Biol Chem 285: 19076-19084, 2010. 
29. Du L, Subauste MC, DeSevo C, et al: miR-337-3p and its targets STAT3 and RAPIA modulate taxane sensitivity in non-small cel lung cancers. PLoS One 7: e39167, 2012.

30. Catuogno S, Cerchia L, Romano G, Pognonec P, Condorelli G and de Franciscis V: miR-34c may protect lung cancer cells from paclitaxel-induced apoptosis. Oncogene 32: 341-351, 2013.

31. Holleman A, Chung I, Olsen RR, et al: miR-135a contributes to paclitaxel resistance in tumor cells both in vitro and in vivo. Oncogene 30: 4386-4398, 2011.

32. Li J, Zhang Y, Zhao J, Kong F and Chen Y: Overexpression of miR-22 reverses paclitaxel-induced chemoresistance through activation of PTEN signaling in p53-mutated colon cancer cells Mol Cell Biochem 357: 31-38, 2011.

33. Zhou M, Liu Z, Zhao Y, et al: MicroRNA-125b confers the resistance of breast cancer cells to paclitaxel through suppression of pro-apoptotic Bcl-2 antagonist killer 1 (Bak1) expression. J Biol Chem 285: 21496-21507, 2010.

34. Mei M, Ren Y, Zhou X, et al: Downregulation of miR-21 enhances chemotherapeutic effect of taxol in breast carcinoma cells. Technol Cancer Res Treat 9: 77-86, 2010.

35. Huang YW, Liu JC, Deatherage DE, et al: Epigenetic repression of microRNA-129-2 leads to overexpression of SOX4 oncogene in endometrial cancer. Cancer Res 69: 9038-9046, 2009.

36. Mialon A, Sankinen M, Soderstrom H, et al: DNA topoisomerase $\mathrm{I}$ is a cofactor for c-Jun in the regulation of epidermal growth factor receptor expression and cancer cell proliferation. Mol Cell Biol 25: 5040-5051, 2005.

37. Barroso-delJesus A, Romero-Lopez C, Lucena-Aguilar G, et al: Embryonic stem cell-specific miR302-367 cluster: human gene structure and functional characterization of its core promoter. Mol Cell Biol 28: 6609-6619, 2008.
38. Lavon I, Zrihan D, Granit A, et al: Gliomas display a microRNA expression profile reminiscent of neural precursor cells. Neuro Oncol 12: 422-433, 2010.

39. Sokilde R, Kaczkowski B, Podolska A, et al: Global microRNA analysis of the NCI-60 cancer cell panel. Mol Cancer Ther 10: 375-384, 2011.

40. Greither T, Grochola LF, Udelnow A, Lautenschlager C, Wurl P and Taubert H: Elevated expression of microRNAs 155, 203, 210 and 222 in pancreatic tumors is associated with poorer survival. Int J Cancer 126: 73-80, 2010.

41. Levati L, Alvino E, Pagani E, et al: Altered expression of selected microRNAs in melanoma: antiproliferative and proapoptotic activity of miRNA-155. Int J Oncol 35: 393-400, 2009.

42. Pan X, Zhao J, Zhang WN, et al: Induction of SOX4 by DNA damage is critical for p53 stabilization and function. Proc Natl Acad Sci USA 106: 3788-3793, 2009.

43. Baraniskin A, Birkenkamp-Demtroder K, Maghnouj A, et al: MiR-30a-5p suppresses tumor growth in colon carcinoma by targeting DTL. Carcinogenesis 33: 732-739, 2012.

44. Yanaihara N, Caplen N, Bowman E, et al: Unique microRNA molecular profiles in lung cancer diagnosis and prognosis. Cancer Cell 9: 189-198, 2006.

45. Marchini S, Cavalieri D, Fruscio R, et al: Association between miR-200c and the survival of patients with stage I epithelial ovarian cancer: a retrospective study of two independent tumour tissue collections. Lancet Oncol 12: 273-285, 2011.

46. Li X, Zhang Y, Zhang Y, Ding J, Wu K and Fan D: Survival prediction of gastric cancer by a seven-microRNA signature. Gut 59: 579-585, 2010. 